\title{
Di-vector Boson Production in Association with Multiple Jets at the LHC
}

\author{
Fernando Febres Cordero, Philipp Hofmann and Harald Ita* \\ Universität Freiburg \\ E-mail: harald.ita@physik.uni-freiburg.de
}

\begin{abstract}
The study of vector-boson pair production in association with jets is an important signature for the analysis of the electroweak symmetry breaking mechanism. While the vector bosons are tags of electroweak dynamics, the associated jets give a handle to suppress backgrounds and probe the kinematic dependence of the interactions. At large jet multiplicity the di-vector boson signature plays a particularly important role as background to Higgs transverse momentum distribution, vector boson scattering, top-quark physics as well as background for searches of new physics. Despite its small cross section, this process class will we accessible by measurements in the ongoing physics run at the LHC with more data and center-of-mass energy available. In this talk we present for the first time next-to-leading order QCD corrections to the Standard Model production of oppositely charged W-bosons in association with up to three jets. The results are obtained by using modern on-shell and unitarity methods, which have been implemented in the BlackHat program. Predictions for total and differential cross sections at the LHC will be presented.
\end{abstract}

The European Physical Society Conference on High Energy Physics

22-29 July 2015

Vienna, Austria

${ }^{*}$ Speaker. 


\section{Introduction}

The expected data sets from present and future LHC runs do not only improve the precision of measurements but also open opportunities to study rare processes. Here we discuss new theory predictions for di-vector boson production in association with multiple jets. In particular, we focus on the production of oppositely charged $W$ bosons including QCD corrections. This signature appears as background to top-quark pair production and measurements of (anomalous) gauge couplings in the weak vector-boson-fusion (VBF) topology. Moreover, this type of signature plays a role in new physics searches including lepton and missing energy final states. Finally, such precise theory predictions are useful per se for improving our understanding of Standard Model (SM) physics.

We consider next-to-leading order (NLO) QCD corrections to the production of oppositely charged vector boson pairs in association with jets. The results for di-vector boson production in association with three jets have not appeared in the literature before. For comparison we discuss as well $W^{+} W^{-}+n$-jet production with $n=0,1$ and 2. We focus on the QCD corrections and do not include sub leading electroweak contributions. In this sense we include contributions from couplings of the vector bosons to light quarks (omitting small third-family-quark loops) as well as tri-linear vector couplings. A first phenomenological study at $8 \mathrm{TeV}$ is presented to assess the impact of NLO QCD corrections on total and differential cross sections at the LHC. We postpone predictions of higher center-of-mass energy to a future publication. In summary we observe that the scale dependence of the theory predictions is improved at NLO in QCD with sensitivity reduced for $W^{+} W^{-}+3$-jet total cross sections from about $38 \%$ at leading order (LO) to $5 \%$ at NLO.

Measurements of the $W W+n$-jet production cross section have played an important role at hadron colliders. Inclusive $W W$ cross sections were first measured by CDF [1] and D0 [2] at the Tevatron, and recently by ATLAS [3, 4] and CMS [5, 6] at the LHC. Only in 2015 a first dedicated measurement of cross sections for $W^{+} W^{-}$production in association with 0,1 and 2 jets has been published [7] (by CDF). For the exploration of the electroweak sector direct $W W+n$-jet signals have been studied. To give a famous example, $W^{+} W^{-}+n$-jet production has been explored in order to reduce this background to Higgs-boson studies with $W^{+} W^{-}$decays $[8,9,10,11]$. Furthermore, last year both ATLAS [12] and CMS [13] published first results for the production of same charge vector-boson scattering in association with two jets. Further dedicated studies of $W^{+} W^{-}+$jets production are expected in the near future. It is the aim of this work to provide reliable SM predictions for such a task.

Over the years, the importance of $W$-pair production has stimulated many theory developments. Early LO results were given in [14] and later NLO QCD corrections added in refs. [15]. Very recently, the first next-to-next-to-leading (NNLO) order QCD prediction [16] was published. Similarly, QCD corrections to $W^{+} W^{-}$production in association with jets are well known by now. The NLO QCD predictions for one jet [17] and two jets [18, 19, 20] are available. In addition, the same sign processes were published in ref. [21]. NLO QCD corrections are particularly large at low jet multiplicity due to the opening of partonic channels. In general, the QCD corrections are moderate and provide reliable predictions for total and many differential cross sections as will be shown below. 


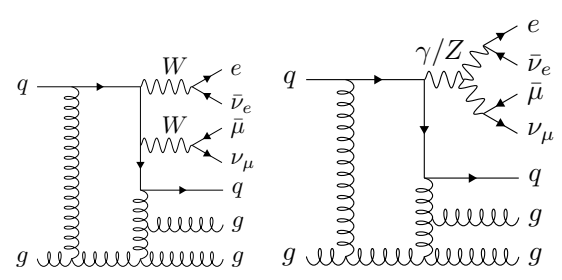

Figure 1: Example diagrams contributing to loop amplitudes of $q g \rightarrow W^{+} W^{-} q^{(\prime)} g g$, followed by the decays $W^{-} \rightarrow e \bar{v}_{e}$ and $W^{+} \rightarrow \bar{\mu} v_{\mu}$. Analogous diagrams are considered for the 4-quark processes. Sub-leading color contributions are small and we omit them here.

\section{Setup}

Predictions at next-to-leading order require the computation of born, real and virtual matrix elements. Given that automated approaches for born and real emissions are available (e.g. [22]) we focus our discussion on the virtual matrix elements. The virtual corrections to $W^{+} W^{-}+3-$ jet production involve up to five final-state objects and include, in addition, the leptonic decay products of the weak vector bosons. Such amplitudes are comparable in complexity to state-of-theart processes with five or more objects in the final state [23, 24, 25, 26].

We use the framework of the BLACKHAT library [27] to compute the one-loop matrix elements. This library uses on-shell methods [28, 29, 30, 31, 32] which construct loop and tree amplitudes from their unitarity and factorization properties. Such methods are important for our computation since their performance scales well as the number of external legs increases. The approach has contributed to NLO QCD predictions to vector-boson production in association with up to five jets [33, 34, 24, 23, 35, 25, 36], pure 4-jet production [37] and di-photon production in association with 2 jets [38].

The functionality of the BLACKHAT library has been extended to provide the virtual matrix elements for QCD corrections to two massive vector bosons. For the unitarity approach this amounts to providing additional tree-level amplitudes and extending the infrastructure of the program to handle them. In order to check our results two approaches are implemented. On the one hand onshell recursion relations [30] for tree amplitudes of quarks, gluons and massive vector bosons [39] (including decay products) have been provided. On the other hand we checked our results using off-shell recursions [40] for tree amplitudes. Once primitive tree and loop amplitudes are available they are assembled into the virtual, squared matrix elements following the algorithm of ref. [41]. The infrastructure in BLACKHAT to perform this step has been extended.

In QCD color degrees of freedom can be used to organise computations into smaller more tractable pieces. The minimal gauge invariant objects that can be isolated in this way are called primitive color ordered amplitudes [42]. In BLACKHAT we first compute these building blocks and assemble them to the full answer in the end. The primitive loop amplitudes can be specified by representative Feynman diagrams which are often referred to as the parent diagrams. The set of all incorporated diagrams is generated from these 'parent diagrams' by collapsing propagators, moving colored legs and pulling trees out of the loops. Parent diagrams for the 2-quark 3-gluon amplitudes are given in Fig. 1. In addition to structuring computations, color degrees of freedom allow to setup an expansion in $1 / N_{c}$-factors assuming a large number of colors $N_{c}$. Although the 


\begin{tabular}{||c||c|c||c|c||}
\cline { 2 - 5 } \multicolumn{1}{c|}{} & \multicolumn{2}{c||}{$W^{+} W^{-}+n$ jet $(8 \mathrm{TeV})$} & \multicolumn{2}{c||}{$R_{n}(8 \mathrm{TeV})$} \\
\hline$n$ & LO & NLO & LO & NLO \\
\hline \hline 0 & $141.7(4)_{-5.3}^{+3.7}$ & $207.9(7)_{-3.5}^{+5.4}$ & - & - \\
\hline 1 & $61.1(2)_{-8.0}^{+9.8}$ & $76.4(4)_{-4.0}^{+3.6}$ & $0.431(2)$ & $0.367(2)$ \\
\hline 2 & $29.44(7)_{-6.92}^{+9.99}$ & $28.8(2)_{-1.9}^{+0.4}$ & $0.482(2)$ & $0.377(3)$ \\
\hline 3 & $11.12(2)_{-3.51}^{+5.74}$ & $9.22(16)_{-1.05}^{+0.17}$ & $0.378(1)$ & $0.320(6)$ \\
\hline 4 & $3.59(2)_{-1.37}^{+2.50}$ & - & $0.323(2)$ & - \\
\hline
\end{tabular}

Table 1: Total cross sections in femtobarns (fb) for $W^{+} W^{-}+n$-jet production at the LHC with $\sqrt{s}=8$ $\mathrm{TeV}$. We show parton-level LO and NLO QCD results, as well as the corresponding jet cross section ratios, $R_{n}=\left(W^{+} W^{-}+n\right.$ jet $) /\left(W^{+} W^{-}+(n-1)\right.$ jet $)$. The NLO result for $W^{+} W^{-}+3$ jets uses the leading-color approximation discussed in the text. In parentheses we show the numerical integration error, and the dependence on the unphysical renormalization and factorization scales is quoted in super and subscripts.

value of $N_{c}$ is set to three in actual computations and $1 / N_{c}$ (or $1 / N_{c}^{2}$ ) is not particularly small, the color expansion typically gives excellent estimates of the full result. One reason for this is that all logarithms appear already in the leading color expressions, thus giving a complete representation of the functional form of the amplitudes. Our numerical results are given in the leading-color approximation where the $1 / N_{c}$-terms are omitted in virtual contributions; all 2-quark as well as 4-quark channels are considered but for each subprocess the approximation is applied. We follow the conventions of ref. [41]; we include virtual quark loops, but omit sub-leading color terms in the partial loop amplitudes. The approximation has been tested for the present process class for $W^{+} W^{-}+1,2$-jet production, where the total cross section is shifted by $1 \%$ when sub-leading color terms are included. The sub-leading color corrections to $W^{+} W^{-}+3$-jet are expected to be similarly small.

The description of the vector-boson final states is adjusted to the di-vector boson production measurement. We consider the double resonant contributions [15] and include the full Breit-Wigner distribution for all intermediate $Z$ and $W$ bosons. The complex mass scheme [43] is used to obtain gauge invariant results. The decays to leptons have all spin correlations included. For simplicity we consider a diagonal Cabibbo-Kobayashi-Maskawa (CKM) matrix. We consider five light quark flavors; $n_{f}=5$. Except for canceling infrared collinear poles, we do not include contribution from external bottom quarks; bottom final states are vetoed. Such final states may be attributed to top production which we do not consider here. Furthermore, we omit initial state bottom quarks due to the sub leading nature of the parton-distribution functions (PDFs). As mentioned above we do not consider the resonant top contributions, which can overwhelm the PDF suppression.

Phase space integration as well as the remaining NLO ingredients, the real-emission and dipole-subtraction terms [44], are computed by COMIX [45] which is part of the SHERPA package [22]. For convenience of the analysis we store intermediate results in public root-format [46] Ntuple files [47], recording the key data of events. The effects of varying scale and input parameters as well as tightening of phase-space cuts are obtained directly from the Ntuple files without recomputing matrix elements.

The results have been checked in a number of ways including the explicit cancellation of 


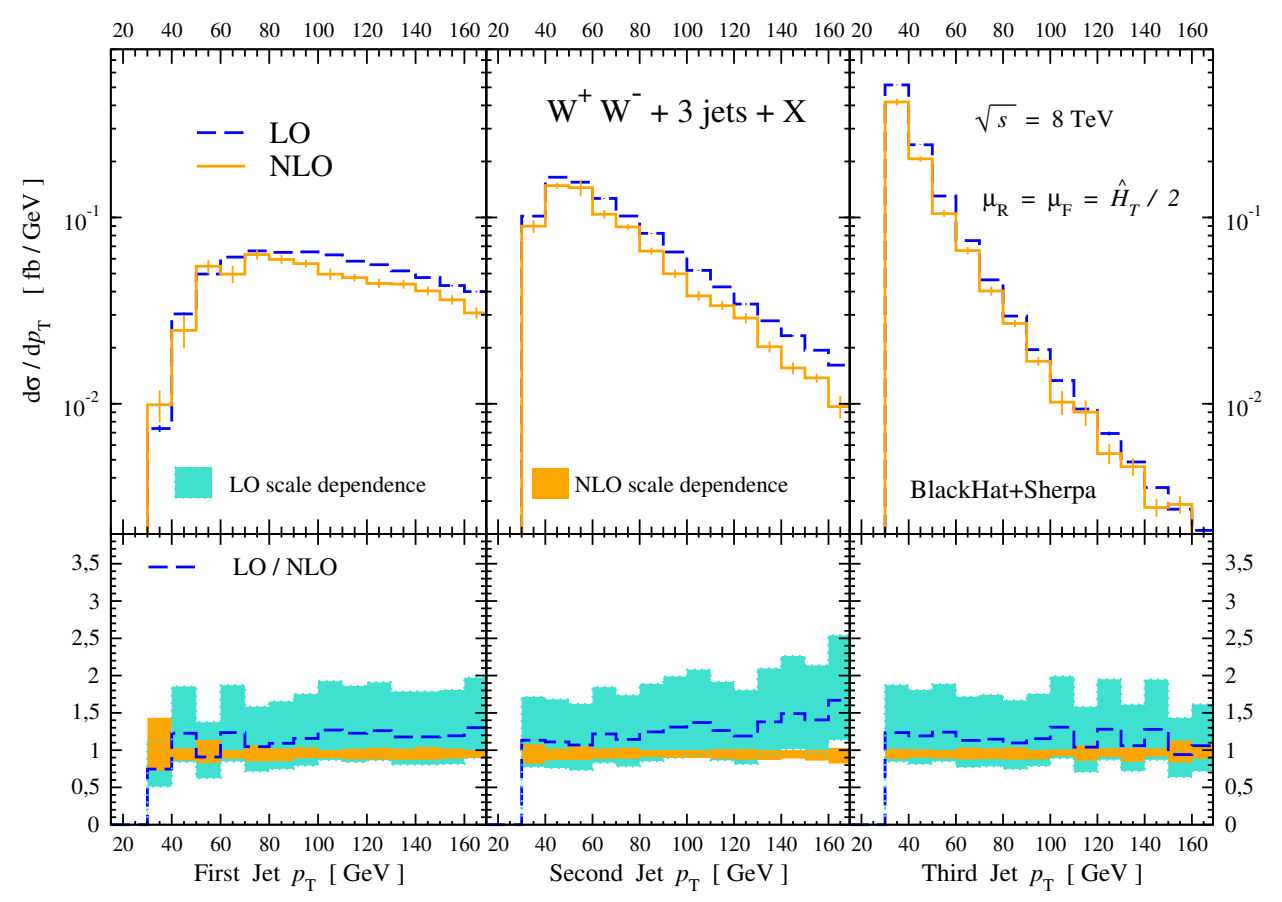

Figure 2: Transverse momentum $p_{T}$ distributions of the jets in $W^{+} W^{-}+3$-jet production for $\sqrt{s}=8 \mathrm{TeV}$. In the upper panels the NLO distribution is the solid (orange) histogram and the LO predictions are shown as dashed (blue) lines. The lower panels show the LO distribution and LO and NLO scale-dependence bands normalized to the central NLO prediction. The bands are dark shaded (orange) for NLO and light shaded (cyan) for LO.

infrared poles and comparison of one-loop matrix elements when available [48]. The SHERPA implementation of the real-emission and dipole-subtraction terms has a dependence on an arbitrary parameter $\alpha_{\text {dipole }}$ [49], and we have made dedicated studies to show that our results are independent of it.

\section{Results}

The inclusive processes $p p \rightarrow W^{+} W^{-}+1,2,3$ jets (also inclusive $W^{+} W^{-}$production) are studied for the LHC setup with proton center-of-mass energy of $\sqrt{s}=8 \mathrm{TeV}$. Results for other center-of-mass energies are available as well and will be made available in a future publication. We also show LO results for $W^{+} W^{-}+4$-jet production. The $W$ bosons are decayed into charged light lepton-neutrino pairs; we require an electron as well as an anti-muon to suppress $Z Z$ production backgrounds.

We consider the following phase-space cuts,

$$
\begin{aligned}
& p_{\mathrm{T}}^{e, \mu}>20 \mathrm{GeV}, \quad\left|\eta^{e, \mu}\right|<2.4, \quad \mathbb{E}_{\mathrm{T}}>30 \mathrm{GeV}, \\
& p_{\mathrm{T}}^{e \mu}>30 \mathrm{GeV} \quad \text { and } \quad m_{e \mu}>10 \mathrm{GeV},
\end{aligned}
$$

with the anti-kt jets [50],

$$
R=0.4 \quad \text { and } \quad p_{T}^{\text {jet }}>30 \mathrm{GeV}, \quad\left|\eta^{\text {jet }}\right|<4.5 .
$$


The standard notation for kinematic variables is used; $p_{T}$ are transverse momenta; $\eta$, pseudo rapidities; $m_{e \mu}$, and $p_{T}^{e \mu}$ the mass and transverse momentum of the electron-muon system, respectively. The total missing energy $\mathbb{E}_{\mathrm{T}}$ is given by the sum of the momenta of the neutrinos pointing into the transverse plane.

At NLO we use the MSTW2008nlo [51] PDFs and the MSTW2008lo set at LO, and we set $\alpha_{s}$ consistently as provided by the PDFs. The $W$ and $Z$ boson mass and width are given respectively by $\Gamma_{W}=2.085 \mathrm{GeV}, M_{W}=80.399 \mathrm{GeV}$ and $\Gamma_{Z}=2.4952 \mathrm{GeV}, M_{W}=91.188 \mathrm{GeV}$. These values, along with $\alpha_{\mathrm{QED}}\left(M_{Z}\right)=1 / 128.802$ are kept fixed for all events.

Compared to LO predictions the NLO cross sections generally have much milder spurious dependence on the setting of the renormalization and factorization scale. Nevertheless, here renormalization and factorization scales are set on an event-by-event basis; we use the total partonic transverse energy which appears representative for the energy transfer of the QCD subprocesses. Explicitly we have $\mu=\mu_{r}=\mu_{f}=\hat{H}_{T} / 2$, where $\hat{H}_{T}=\sum_{j} p_{T}^{j}$ and the sum runs over all final-state partons and leptons $j$. The scale-setting dependence is probed by the standard procedure of varying the chosen central scale up and down by a factor of two. The scale variation bands are presented in the bottom panel of fig. 2

In table 1 , we present LO and NLO parton-level cross sections at $\sqrt{s}=8 \mathrm{TeV}$ and include as well LO results for $W^{+} W^{-}+4$ jets. The scale-variation for the NLO cross sections amount to $5 \%$ for our setup as opposed to the LO values which vary from $7 \%$ to $50 \%$ for zero up to four jets at $8 \mathrm{TeV}$.

A further interesting quantity are the "jet-production" ratios of $W^{+} W^{-}+n$-jet to $W^{+} W^{-}+(n-1)$ jet production. These ratios are less sensitive to experimental and theoretical systematics than the absolute cross sections. Despite a significant dependence of the ratios on the phase-space cuts imposed [52] universal behavior of these ratios can be expected for large jet multiplicities in sufficiently hard regions of phase space. Further studies about these ratios will be performed in the future.

In Fig. 2, we show the $p_{T}$ spectra of the leading three jets in $W^{+} W^{-}+3$-jet production at LO and NLO at $\sqrt{s}=8 \mathrm{TeV}$; in the lower panels we normalize (bin-by-bin) to the NLO prediction and show scale-dependence bands. We observe a noticeable shape difference between the LO and NLO distributions for the first two leading jets, while the shapes for the third-jet distribution is very similar at LO and NLO. Similar behavior has been observed in $W$ production in association with jets [23]. We show the NLO and LO predictions, along with their scale-dependence bands. In the $p_{T}$ distributions the NLO band is reduced in size giving an estimate of the theoretical uncertainty below the ten per cent level.

\section{Conclusion}

The presented scattering process is the first in a new class of predictions, that is di-vector boson production in association with three jets.

Already with the present results a number of immediate phenomenological questions can be addressed. It will be interesting to increase the collision energy and, furthermore, to study the effect of QCD corrections when requiring two forward tagging jets which emphasize weak vector-bosonfusion event topologies. Moreover, it will be interesting to compute corresponding corrections in 
scenarios relevant for new-physics searches.

Extending the $W W+n$-jet processes, additional combinations of vector-boson pairs $V V^{\prime}$ (with $V$ and $V^{\prime}$ either $W^{ \pm}, Z$ or a photon) as well as interference effects with electroweak processes can be considered. This class of processes plays an important role for exploring the electroweak symmetry breaking mechanism. These processes are well within reach of the current BLACKHAT library and we expect to provide new theory prediction for this process class in the near future.

Acknowledgements: We thank Z. Bern, L. Dixon, D. Kosower, D. Maître and C. Schwinn for helpful discussions. The work of F.F.C. is supported by the Alexander von Humboldt Foundation, in the framework of the Sofja Kovalevskaja Award 2014, endowed by the German Federal Ministry of Education and Research. P.H.'s work is supported by the Juniorprofessor Program of Ministry of Science, Research and the Arts of the state of Baden-Württemberg, Germany. H.I.'s work is supported by a Marie Skłodowska-Curie Action Career-Integration Grant PCIG12-GA-2012-334228 of the European Union. This work was performed on the bwUniCluster funded by the Ministry of Science, Research and the Arts Baden-Württemberg and the Universities of the State of BadenWürttemberg, Germany, within the framework program bwHP, as well as using the computational and storage services associated with the Hoffman2 Shared Cluster provided by UCLA Institute for Digital Research and Education's Research Technology Group.

\section{References}

[1] T. Aaltonen et al. (CDF Collaboration), Phys. Rev. Lett. 104, 201801 (2010) .

[2] V. Abazov et al. (D0 Collaboration), Phys. Rev. Lett. 103, 191801 (2009) .

[3] ATLAS Collaboration, Phys. Rev. Lett. 107, 041802 (2011).

[4] ATLAS Collaboration, Phys. Rev. D 87, no. 11, 112001 (2013) [Phys. Rev. D 88, no. 7, 079906 (2013)] .

[5] CMS Collaboration, Eur. Phys. J. C 73, no. 10, 2610 (2013).

[6] CMS Collaboration, Phys. Lett. B 721, 190 (2013).

[7] T. A. Aaltonen et al. [CDF Collaboration], Phys. Rev. D 91 (2015) 11, 111101 [Phys. Rev. D 92 (2015) 3, 039901].

[8] T. Aaltonen et al. [CDF Collaboration], Phys. Rev. D 88, no. 5, 052012 (2013) .

[9] V. M. Abazov et al. [D0 Collaboration], Phys. Rev. D 88, no. 5, 052006 (2013) .

[10] CMS Collaboration, JHEP 1401, 096 (2014) .

[11] ATLAS Collaboration, arXiv:1412.2641 [hep-ex].

[12] ATLAS Collaboration, Phys. Rev. Lett. 113, no. 14, 141803 (2014) .

[13] CMS Collaboration, Phys. Rev. Lett. 114, no. 5, 051801 (2015) .

[14] R. W. Brown and K. O. Mikaelian, Phys. Rev. D 19, 922 (1979).

[15] J. Ohnemus, Phys. Rev. D 44, 1403 (1991); S. Frixione, Nucl. Phys. B 410, 280 (1993); L. J. Dixon, Z. Kunszt and A. Signer, Nucl. Phys. B 531 (1998) 3 ; J. M. Campbell and R. K. Ellis, Phys. Rev. D 60, 113006 (1999) ; L. J. Dixon, Z. Kunszt and A. Signer, Phys. Rev. D 60, 114037 (1999) ; J. M. Campbell, R. K. Ellis and C. Williams, JHEP 1107, 018 (2011). 
[16] T. Gehrmann, M. Grazzini, S. Kallweit, P. Maierhöfer, A. von Manteuffel, S. Pozzorini, D. Rathlev and L. Tancreedi, Phys. Rev. Lett. 113, no. 21, 212001 (2014) .

[17] J. M. Campbell, R. K. Ellis and G. Zanderighi, JHEP 0712, 056 (2007) ; S. Dittmaier, S. Kallweit and P. Uwer, Phys. Rev. Lett. 100, 062003 (2008) ; S. Dittmaier, S. Kallweit and P. Uwer, Nucl. Phys. B 826, 18 (2010) ; J. M. Campbell, D. J. Miller and T. Robens, Phys. Rev. D 92 (2015) 1, 014033.

[18] T. Melia, K. Melnikov, R. Rontsch and G. Zanderighi, Phys. Rev. D 83, 114043 (2011) .

[19] N. Greiner, G. Heinrich, P. Mastrolia, G. Ossola, T. Reiter and F. Tramontano, Phys. Lett. B 713, 277 (2012) .

[20] J. Alwall, R. Frederix, S. Frixione, V. Hirschi, F. Maltoni, O. Mattelaer, H.-S. Shao and T. Stelzer et al., JHEP 1407 (2014) 079.

[21] T. Melia, K. Melnikov, R. Rontsch and G. Zanderighi, JHEP 1012, 053 (2010) ; F. Campanario, M. Kerner, L. D. Ninh and D. Zeppenfeld, Phys. Rev. D 89, no. 5, 054009 (2014) .

[22] T. Gleisberg, S. Höche, F. Krauss, M. Schönherr, S. Schumann, F. Siegert and J. Winter, JHEP 0902, 007 (2009).

[23] Z. Bern, L. J. Dixon, F. Febres Cordero,S. Höche, H. Ita, D. A. Kosower ,D. Maître and K. J. Ozeren Phys. Rev. D 88, no. 1, 014025 (2013).

[24] C. F. Berger, Z. Bern, L. J. Dixon, F. Febres Cordero, D. Forde, T. Gleisberg, H. Ita and D. A. Kosower, D. Maître, Phys. Rev. Lett. 106, 092001 (2011)

[25] H. Ita, Z. Bern, L. J. Dixon, F. Febres Cordero, D. A. Kosower and D. Maître, Phys. Rev. D 85, 031501 (2012).

[26] S. Badger, B. Biedermann, P. Uwer and V. Yundin, Phys. Rev. D 89, no. 3, 034019 (2014) ; S. Badger, A. Guffanti and V. Yundin, JHEP 1403, 122 (2014); A. Denner and R. Feger, arXiv:1506.07448 [hep-ph].

[27] C. F. Berger, Z. Bern, L. J. Dixon, F. Febres Cordero, D. Forde, H. Ita, D. A. Kosower and D. Maître Phys. Rev. D 78, 036003 (2008).

[28] Z. Bern, L. J. Dixon, D. C. Dunbar and D. A. Kosower, Nucl. Phys. B 425, 217 (1994); Nucl. Phys. B 435, 59 (1995); Z. Bern, L. J. Dixon and D. A. Kosower, Nucl. Phys. B 513, 3 (1998).

[29] R. Britto, F. Cachazo and B. Feng, Nucl. Phys. B 725, 275 (2005).

[30] R. Britto, F. Cachazo, B. Feng and E. Witten, Phys. Rev. Lett. 94 (2005) 181602.

[31] G. Ossola, C. G. Papadopoulos and R. Pittau, Nucl. Phys. B 763, 147 (2007); D. Forde, Phys. Rev. D 75, 125019 (2007); W. T. Giele, Z. Kunszt and K. Melnikov, JHEP 0804, 049 (2008); S. D. Badger, JHEP 0901, 049 (2009).

[32] Z. Bern, L. J. Dixon and D. A. Kosower, Annals Phys. 322, 1587 (2007); C. F. Berger and D. Forde, Ann. Rev. Nucl. Part. Sci. 60, 181 (2010); H. Ita, J. Phys. A 44 (2011) 454005.

[33] C. F. Berger, Z. Bern, L. J. Dixon, F. Febres Cordero, D. Forde, T. Gleisberg, H. Ita, D. A. Kosower and D. Maître, Phys. Rev. Lett. 102, 222001 (2009).

[34] C. F. Berger, Z. Bern, L. J. Dixon, F. Febres Cordero, D. Forde, T. Gleisberg, H. Ita, D. A. Kosower and D. Maître, Phys. Rev. D 80, 074036 (2009).

[35] C. F. Berger, Z. Bern, L. J. Dixon, F. Febres Cordero, D. Forde, T. Gleisberg, H. Ita, D. A. Kosower and D. Maître, Phys. Rev. D 82, 074002 (2010). 
[36] Z. Bern, G. Diana, L. J. Dixon, F. Febres Cordero, S. Höche, H. Ita, D. A. Kosower and D. Maître and K. J. Ozeren, Phys. Rev. D 84, 114002 (2011), Phys. Rev. D 87, no. 3, 034026 (2013) .

[37] Z. Bern, G. Diana, L. J. Dixon, F. Febres Cordero, S. Höche, D. A. Kosower, H. Ita, D. Maitre and K. Ozeren, Phys. Rev. Lett. 109, 042001 (2012) .

[38] Z. Bern, L. J. Dixon, F. Febres Cordero, S. Höche, H. Ita, D. A. Kosower, N. A. Lo Presti and D. Maitre, Phys. Rev. D 90, 054004 (2014).

[39] S. D. Badger, E. W. N. Glover and V. V. Khoze, JHEP 0601 (2006) 066.

[40] F. A. Berends and W. T. Giele, Nucl. Phys. B 306 (1988) 759.

[41] H. Ita and K. Ozeren, JHEP 1202, 118 (2012) .

[42] Z. Bern, L. J. Dixon and D. A. Kosower, Ann. Rev. Nucl. Part. Sci. 46 (1996) 109.

[43] A. Denner, S. Dittmaier, M. Roth and D. Wackeroth, Nucl. Phys. B 560 (1999) 33.

[44] S. Catani and M. H. Seymour, Nucl. Phys. B 485, 291 (1997) [Erratum-ibid. B 510, 503 (1998)].

[45] T. Gleisberg and S. Höche, JHEP 0812, 039 (2008) .

[46] R. Brun and F. Rademakers, Nucl. Instrum. Meth. A 389, 81 (1997).

[47] Z. Bern, L. J. Dixon, F. Febres Cordero, S. Höche, H. Ita, D. A. Kosower and D. Maitre, Comput. Phys. Commun. 185, 1443 (2014) .

[48] G. Cullen, H. van Deurzen, N. Greiner, G. Heinrich, G. Luisoni, P. Mastrolia, E. Mirabella and G. Ossola et al., Eur. Phys. J. C 74, no. 8, 3001 (2014) .

[49] Z. Nagy, Phys. Rev. D 68, 094002 (2003).

[50] M. Cacciari, G. P. Salam and G. Soyez, JHEP 0804, 063 (2008).

[51] A. D. Martin, W. J. Stirling, R. S. Thorne and G. Watt, Eur. Phys. J. C 63, 189 (2009).

[52] Z. Bern, L. J. Dixon, F. Febres Cordero, S. Höche, H. Ita, D. A. Kosower and D. Maître, Phys. Rev. D 92 (2015) 1, 014008 